\title{
Magnetic Clouds and Force-Free Fields With Constant Alpha
}

\author{
L. F. Burlaga \\ Laboratory for Extraterrestrial Physics, NASA Goddard Space Flight Center, Greenbelt, Maryland
}

\begin{abstract}
Magnetic clouds observed at $1 \mathrm{AU}$ are modeled as cylindrically symmetric, constant alpha force-free magnetic fields. The model satisfactorily explains the types of variations of the magnetic field direction that are observed as a magnetic cloud moves past a spacecraft in terms of the possible orientations of the axis of a magnetic cloud. The model also explains why the magnetic field strength is observed to be higher inside a magnetic cloud than near its boundaries. However, the model predicts that the magnetic field strength profile should be symmetric with respect to the axis of the magnetic cloud, whereas observations show that this is not generally the case.
\end{abstract}

\section{INTRODUCTION}

A magnetic cloud was identified by Burlaga et al. [1981] as an interplanetary structure with a dimension of the order of $0.25 \mathrm{AU}$ in which the magnetic field strength is higher than average, the magnetic field direction rotates monotonically through a large angle, the temperature is low, and the plasma beta is significantly lower than 1 . Observations concerning the nature, origin, and evolution of magnetic clouds were reviewed by Burlaga [1984]. Burlaga et al. [1981] and Klein and Burlaga [1982] considered some possible magnetic field configurations in magnetic clouds, and in particular they considered the possibility that magnetic clouds might be similar to the Bennett pinch configuration in which the magnetic field lines are a family of circles centered about the axis of the magnetic cloud or to a family of tightly wound helices. Models of pinch configurations were constructed by Suess [1988]. The failure to observe low magnetic field intensities in magnetic clouds, the instability of the pinch configuration, and the general incompatibility of the observed magnetic field patterns with the pinch configuration led Burlaga and Behannon [1982] to consider other configurations that were consistent with the observations, but they did not arrive at a unique result. A quantitative model of a more general type of magnetic cloud was presented by Ivanov and Harshiladze [1984] based on earlier ideas of Parker [1957], but no attempt was made to fit the observations.

The idea that magnetic clouds are force-free configurations was first published by Goldstein [1983], who argued qualitatively that the pattern of magnetic field directions associated with the motion of a magnetic cloud past a spacecraft that was drawn by Burlaga and Behannon [1982] could be produced by a cylindrically symmetric force-free magnetic field configuration with variable alpha in which the magnetic field lines are a family of helices. The idea that force-free fields might be observed on the Sun and in astrophysical plasmas was introduced by Lust and Schluter [1954].

In general, a force-free configuration is given by the equation curl $\mathbf{B}=\alpha \mathbf{B}$, where $\alpha$ is a function of position, and there is an infinite number of configurations corresponding to the possible choices of $\alpha$. "Force free" means that the Lorentz force vanishes; however, one can regard a force-

This paper is not subject to U.S. copyright. Published in 1988 by the American Geophysical Union.

Paper number 7A9313. free configuration as one in which the magnetic pressure is balanced by the tension of the curved magnetic field lines [Ferraro and Plumpton, 1966]. Three general ways to specify a force-free configuration are discussed by Hood [1985]. Goldstein [1983] suggested the use of cylindrical symmetry and an arbitrary generating function, following Lust and Schluter [1954], but he did not write down a particular solution which might describe the observations. Marubashi [1986] assumed cylindrical symmetry and chose a particular variation of the pitch angle $\omega$ with distance $R$ from the axis of a cylindrical magnetic cloud, namely, $\omega \sim R^{2}$, and he obtained good fits to the observations of two magnetic clouds in this way. The approaches of Goldstein and Marubashi are unsatisfactory insofar as they involve an arbitrary choice of some function.

A simple solution for a cylindrically symmetric force-free field with constant alpha was found by Lundquist [1950], and many subsequent studies have shown that constant alpha force-free fields are very special and important configurations. Taylor $[1974,1976,1986]$ conjectured that in a plasma with a finite resistivity, however small, confined to a volume bounded by perfectly conducting walls, the helicity over the whole volume is constant, and the system evolves by turbulent relaxation to a constant alpha force-free configuration. This concept has been applied to astrophysical jets (Konigl and Choudhur [1985]; but see Turner [1986]) and to solar magnetic fields [Heyvaerts and Priest, 1984].

By minimizing the magnetic energy subject to the constraint of constant magnetic helicity, Woltjer [1958] has shown that a force-free field with constant alpha represents the state of lowest magnetic energy which a closed ideal magnetohydrodynamic (MHD) system may attain. He claimed that such a system is stable, and he suggested without proof that it represents the natural end configuration of a system with dissipation. The stability of constant alpha force-free configurations was discussed from a different point of view by Chandrasekhar and Woltjer [1958].

On the other hand, it is not clear that a constant alpha force-free configuration is stable. Voslamber and Callebaut [1962] showed that a force-free field with constant alpha is unstable to kink-type perturbations. However, the characteristic wavelength is several times the cylinder radius, and the growth time of the instability is much larger than the transit time to $1 \mathrm{AU}$ in the case of a magnetic cloud. Force-free fields with nonreversed axial field are believed to be MHD unstable because of a minimum in the $q$ profile [Taylor, 1976]. 
Moreover, it is not clear that the limit of zero pressure and resistivity is the same as the case of precisely zero pressure and resistivity which was considered by Lundquist. Field [1986] has derived the equation for magnetostatic equilibrium using a variational principle which includes the internal energy associated with thermal pressure, and he observed that in the limit $p \rightarrow 0$ one recovers the force-free field equation, but there is no implication that alpha is constant. Since the thermal pressure in magnetic clouds is small but not zero, Woltjer's argument and Lundquist's solution need not apply to magnetic clouds if Field is correct. Turner and Prager [1987] showed that the constant alpha force-free configuration is not a steady state in the presence of resistive dissipation, no matter how small. However, it is a minimum energy state when the resistivity is zero, and that is the mean configuration of a temporally varying state when the resistivity is finite. Using a three-dimensional incompressible MHD code, Dahlburg et al. [1986] showed that a turbulent pinch does tend to relax to a force-free configuration with nonconstant alpha, but it is not clear that magnetic clouds evolve in this way.

Magnetic clouds are of finite extent, they have a resistivity which is vanishingly small, and they have a small but nonzero thermal pressure. They are immersed in an infinitely conducting plasma in which the magnetic pressure is of the order of the magnetic pressure in the magnetic cloud. In general, the magnetic cloud may be bounded by a current sheet, but a systematic study of the boundaries of magnetic clouds has not been made. In the absence of such a boundary there could be no force-free magnetic cloud, since as a result of the virial theorem there are no force-free magnetic fields, other than $B=0$, for which $\mathbf{J}$ is confined to a finite volume and $B$ is everywhere differentiable [Moffatt, 1978, p. 28]. There is at present no theory of how a magnetic cloud forms, and in particular there is no theory of how a magnetic cloud might evolve to a force-free configuration. Moreover, there is no stability analysis of a constant alpha configuration for a free boundary in a compressible plasma. Thus we have no bias for predicting that a magnetic cloud should be a stable constant alpha force-free configuration. Nevertheless, it is reasonable to ask whether Lundquist's solution can provide a description of magnetic clouds, in view of the success of Marubashi's model and given that much is known about constant alpha force-free configurations.

The aim of this paper is simply to determine whether or not Lundquist's solution for a cylindrically symmetric forcefree magnetic field with constant alpha (given in section 2) can describe the types of signatures observed in the solar wind at $1 \mathrm{AU}$ when magnetic clouds move past a spacecraft. In section 3 it is shown that Lundquist's solution does describe several types of variation of the magnetic field direction observed in magnetic clouds, but it is less successful in describing the variation of the magnetic field strength. Our result provides a convenient means of summarizing the observations of magnetic clouds, and it suggests a method of determining the local orientation of a magnetic cloud. The observed connection between magnetic clouds and constant alpha force-free magnetic fields (bearing in mind its limitations) raises a number of theoretical questions concerning the stability, formation, and evolution of magnetic clouds and empirical questions concerning the structure, boundary, origin, and evolution of magnetic clouds, which are discussed briefly in section 4 .
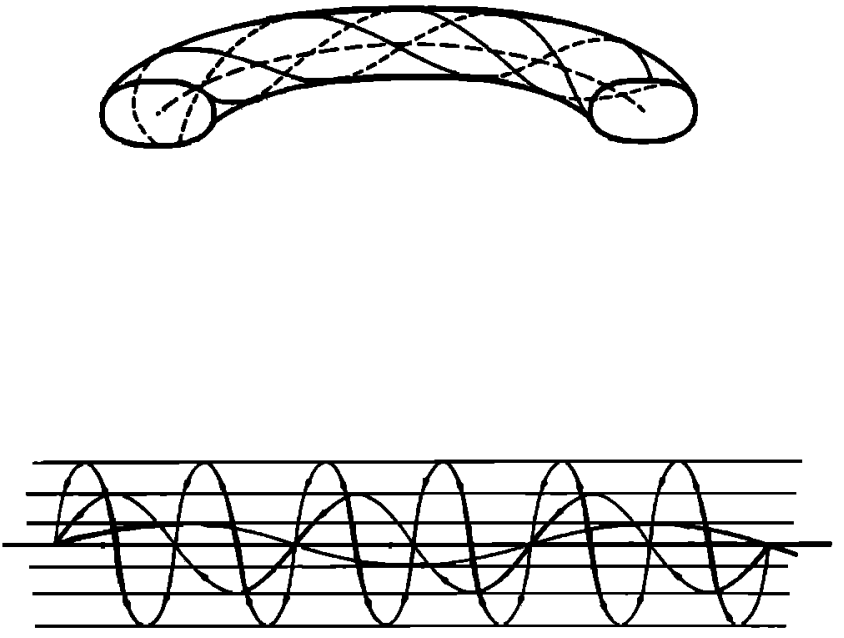

Fig. 1. (Bottom) The magnetic field lines for a cylindrically symmetric constant alpha force-free magnetic field are a family of helices which are shown here projected onto the plane of the paper. (Top) Although magnetic clouds may be locally cylindrically symmetric, they must be curved on a larger scale as illustrated here.

\section{Model of Magnetic Clouds}

Let us assume that locally a magnetic cloud has cylindrical symmetry and apply the solution for a force-free configuration with constant alpha given by Lundquist [1950], namely,

$$
B_{A}=B_{0} J_{0}(a R) \quad B_{T}=B_{0} H J_{1}(a R) \quad B_{R}=0
$$

where $B_{A}$ is the component of the magnetic field along the axis of the magnetic cloud, $B_{T}$ is the component of the magnetic field in the azimuthal direction, $B_{R}$ is the radial component of the magnetic field, $J_{0}$ and $J_{1}$ are Bessel functions, $R$ is the distance from the axis, $H= \pm 1$ determines the handedness of the magnetic field, and $B_{0}$ and $a$ are constants. The solutions for a spherically symmetric magnetic cloud [Chandrasekhar and Kendall, 1957] and a toroidal magnetic cloud [Buck, 1965] are also available, but their application to the solar wind will not be considered here. The lines of force of the magnetic field given by (1) are a family of helices with pitch angle increasing from the axis of the magnetic cloud (where the magnetic field is a straight line) to the boundary (where the lines of force are circles), as illustrated by the drawing at the bottom of Figure 1, which shows a projection of these helices in a plane [Ferraro and Plumpton, 1966]. Configurations of magnetic field lines of this form have been identified in the ionosphere of Venus [Elphic and Russell, 1983], but the model which they introduced is different from the force-free configuration. It would be of interest to determine whether or not the constant alpha force-free solution is also applicable to the flux ropes observed at Venus and Earth, but this will not be considered here.

In reality, a magnetic cloud cannot extend to infinity; hence it must be curved as indicated at the top of Figure 1. It is not known whether the ends of the magnetic cloud are anchored at the Sun or detached from the Sun and joined together to form a torus.

The data that will be considered below are hour averages of the magnetic field from the experiments of Ness and Lepping on the IMP spacecraft and Smith on ISEE 3. The magnetic field is given in solar ecliptic coordinates, where the $X$ axis points from the Earth to the Sun, the $Z$ axis is 


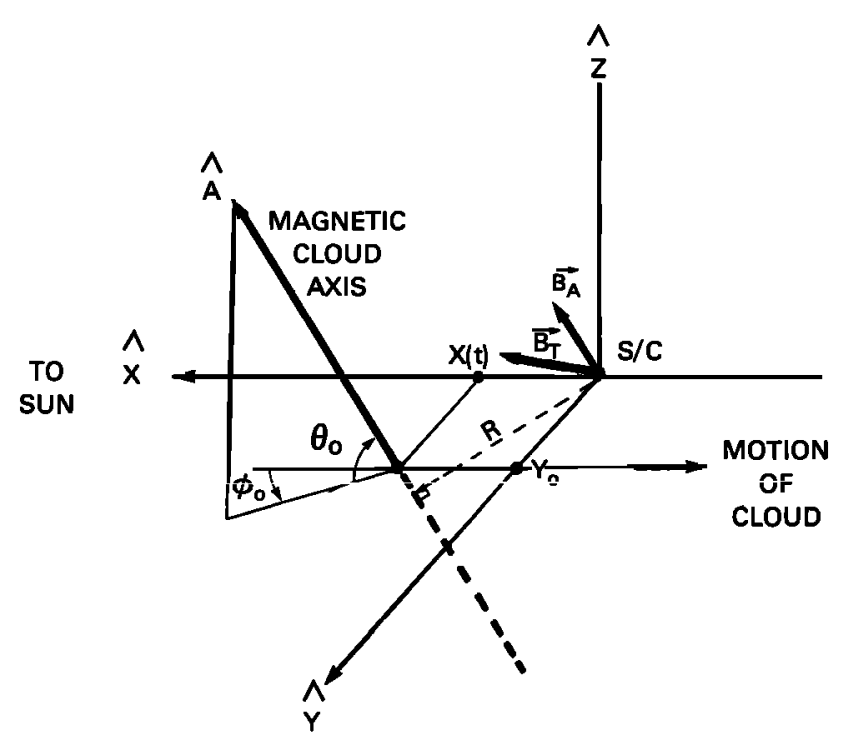

Fig. 2. The geometry which is assumed for the computation of the magnetic field profile that is observed when a magnetic cloud moves past a spacecraft.

normal to the ecliptic, and the $Y$ axis forms a right-handed coordinate system (see Figure 2). Let us assume that magnetic clouds move radially away from the Sun at constant speed during their motion past a spacecraft. Let the orientation of the magnetic cloud be given by the direction of its axis, $\left(\theta_{0}, \phi_{0}\right)$, and let the position of the magnetic cloud be given by the intersection of the axis with the ecliptic plane, $\left(X_{0}(t), Y_{0}\right)$, where $Y_{0}$ is a constant (see Figure 2).

The magnitude of the magnetic field at any instant is $B=$ $\left(B_{A}{ }^{2}+B_{T}^{2}\right)^{1 / 2}$, which decreases from a maximum $B_{\max }$ on the axis of the magnetic cloud to $\sim 0.5 B_{\max }$ at the outer boundary for the magnetic field given by (1). In order to compare the theoretical magnetic field direction with the observations, it is necessary to express the magnetic field in solar ecliptic coordinates for a magnetic cloud of a given orientation $\hat{A}$, position $\left(X(t), Y_{0}, 0\right)$, and handedness $(H=$ \pm 1 ). Figure 2 shows the geometry and the directions of $\mathbf{B}_{A}$ and $\mathbf{B}_{T}$ at the spacecraft at the time $t$. The direction of the magnetic field may be found by resolving $B_{A}$ (which is along $\hat{A}$ ) and $\mathbf{B}_{T}$ (which is normal to the plane defined by $\hat{A}$ and $\mathbf{R}$ ) along $\hat{X}, \hat{Y}, \hat{Z}$ and computing $\theta=\sin ^{-1}\left(B_{Z} / B\right), \phi=\tan ^{-1}$ $\left(B_{Y}\left(B_{X}\right)\right.$.

The general types of profiles that one can expect to see when a magnetic cloud moves past a spacecraft may be illustrated by considering three cases: case 1 , with $\theta_{0}=0^{\circ}$, $\phi_{0}=90^{\circ}$, corresponding to a magnetic cloud whose axis is in the ecliptic and at right angles to the Earth-Sun line; case 2, with $\theta_{0}=90^{\circ}, Y_{0}=1.5$, corresponding to a magnetic cloud whose axis is normal to the ecliptic and at some distance from the spacecraft; and case 3 , with $\theta_{0}=45^{\circ}, \phi_{0}=135^{\circ}$, and $Y_{0}=1$, which is intermediate between case 1 and case 2 .

Case 1. The magnetic field profile that one expects to observe during the passage of a constant alpha force-free magnetic cloud whose axis is in the ecliptic and normal to the Earth-Sun line is shown in Figure $3 a$. The enhanced magnetic field strength and the monotonic variation of the magnetic field from south to north (or from north to south) are characteristic features of a magnetic cloud. The angle $\theta$ increases from $-90^{\circ}$ to $90^{\circ}$ in the main body of the magnetic cloud, and the profile of theta is antisymmetric with respect to $X=0$. The $\phi$ angle is constant in the main body of the magnetic cloud, because $B_{x}=0$. The ratio of the maximum magnetic field strength to the asymptotic value on the boundary (where, by our definition, the magnetic field direction is normal to the axis of the magnetic cloud and $B_{A}=0$ ) is $\sim 0.5 B_{\max }$.

Burlaga et al. [1981] defined the boundaries of a magnetic cloud to be the points at which the elevation angles of the magnetic field are extremal. This definition has the advantage of being unambiguous in an operational sense, but it is not based on physical considerations. Figure $3 a$ suggests that the boundary might extend beyond these limits if the $\phi$ component of the magnetic field reverses near the outer boundary. Although one should keep in mind the possibility of this effect, it is not the primary feature of a magnetic cloud, and the definition of Burlaga et al. [1981] certainly includes the principal part of a magnetic cloud. We show the boundaries as the points where $B_{A}=0$, i.e., where $a R=2.4$ and $B / B_{0}=0.5$.

Case 2. When the axis of the magnetic cloud is normal to the ecliptic and the shortest distance between the spacecraft and the axis of the magnetic cloud is $Y_{0}=1.5$, the magnetic field profile is that shown in Figure $3 b$. In this case, $\phi$ shows a monotonic variation through $180^{\circ}$ in the main body of the magnetic cloud, and $\theta$ is symmetrical about $X=0$. This is not the typical form for a magnetic cloud at $1 \mathrm{AU}$, but it will be shown below that such a form does occur. The magnetic field strength is enhanced in the magnetic cloud, but $B_{\max } / B_{0}$ is significantly less than 1 , because the axis of the magnetic cloud did not pass close to the spacecraft.

Case 3. When the axis of the magnetic cloud is inclined $45^{\circ}$ with respect to the ecliptic and its projection in the ecliptic is inclined $45^{\circ}$ with respect to the Earth-Sun line, the magnetic field profile of a magnetic cloud with $Y_{0}=1$ is that shown in Figure 4. In this case the angles $\theta$ and $\phi$ and the magnetic field strength profile are not symmetric with respect to $X=0$, but the profile may be viewed as a distortion of the profile in Figure $3 b$ which is tending toward the profile in Figure $3 a$. The maximum magnetic field strength for the case in which $Y=1.0$ is intermediate between that for the cases $Y=0$ and $Y=1.5$ shown in Figures $3 a$ and $3 b$.

\section{Fits to Observations of Magnetic Clouds}

In this section it will be shown that the constant alpha force-free magnetic field model of magnetic clouds can describe four types of magnetic field profiles observed during the motion of magnetic clouds past a spacecraft at $1 \mathrm{AU}$. Our choices of the $\theta, \phi$, and $B$ were made by trial and error, so that our fits are not optimal. In subsequent studies it may be desirable to devise a scheme for fitting the data to the model (1). The aim here is to show qualitatively that the model can produce the types of configurations that are observed. Quantitative models will have to address other factors, such as the interaction of the magnetic cloud with the medium ahead or with flows advancing from behind, the nonuniform speed profile within a magnetic cloud, the external total pressure, the internal plasma pressure, departures from cylindrical symmetry, expansion of the magnetic cloud, etc.

Marubashi has modeled two magnetic clouds using a cylindrically symmetric force-free magnetic field model with a variable alpha corresponding to a pitch angle of the magnetic field which increases as the square of the distance from the axis of the magnetic cloud. Let us consider how 


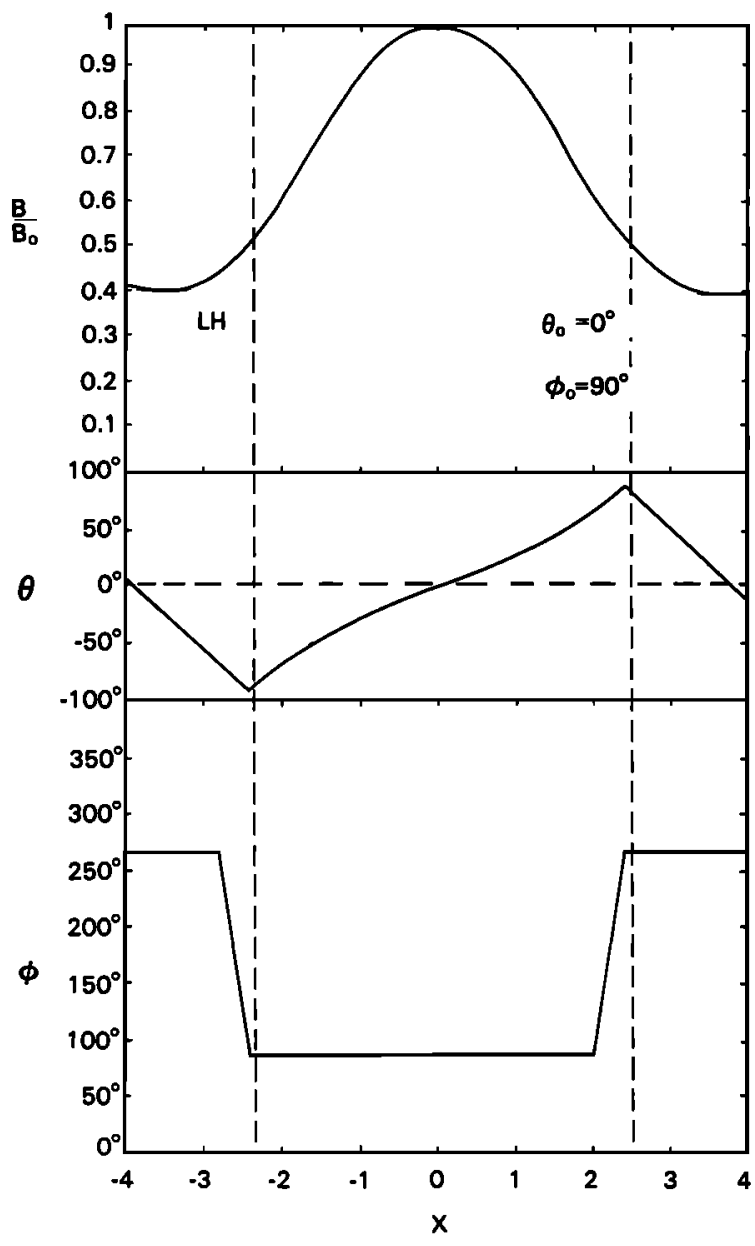

(a)

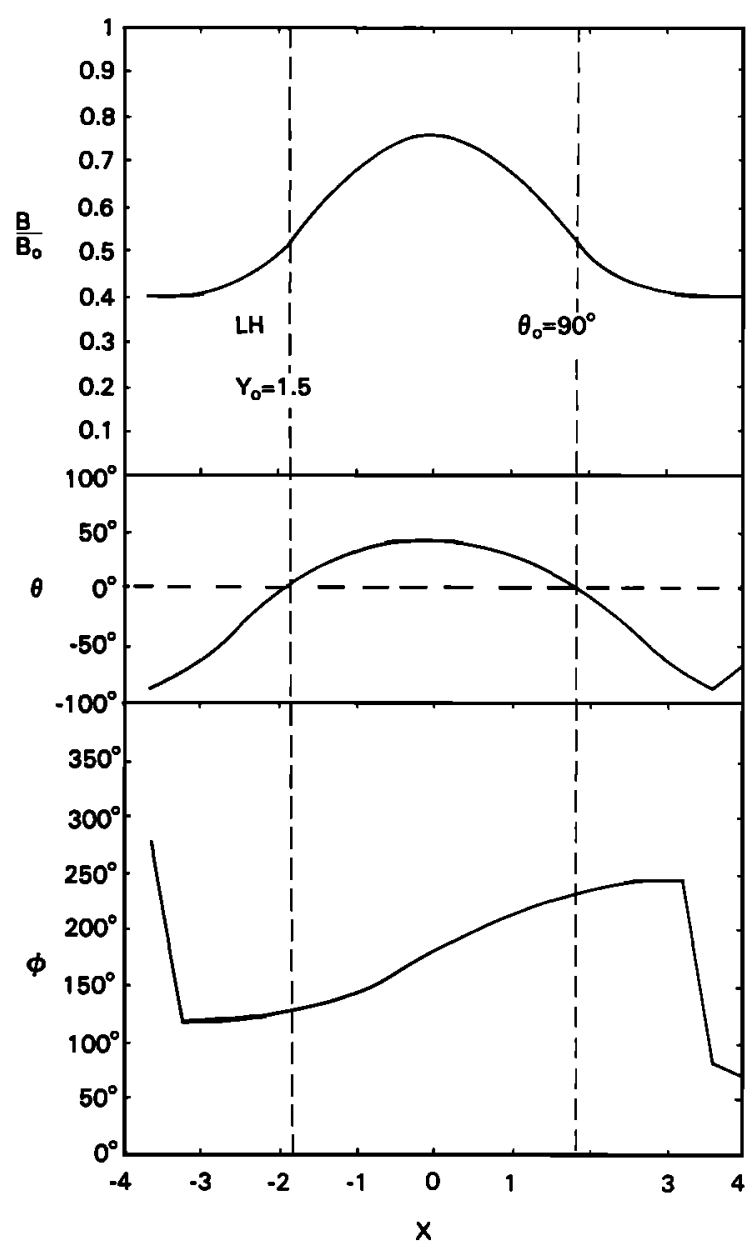

(b)

Fig. 3. (a) The theoretical magnetic field profile which would be observed for a left-handed magnetic cloud whose axis is in the ecliptic and normal to the Earth-Sun line. $(b)$ The theoretical magnetic field profile which would be observed for a left-handed magnetic cloud whose axis is normal to the ecliptic and whose closest approach to the Earth is $Y_{0}=1.5$.

well the constant alpha force-free magnetic field model describes the data for those events, namely, December 29-30, 1967, and June 23, 1971.

The data for the June 23, 1971, event are shown in Figure 5 together with a solution for the constant alpha force-free field. The parameters used for the model are the same as those used by Marubashi in his model: $\theta_{0}=60^{\circ}, \phi_{0}=75^{\circ}$, $Y_{0}=0.2$, and left-hand orientation. The constant alpha solution provides a very satisfactory fit to the magnetic field direction as a function of time during the passage of the magnetic cloud. The model profile for the magnetic field intensity as a function of time does not agree very well with the observed intensity profile, however. Marubashi found a similar discrepancy, which he interpreted as an indication that the force-free condition is not satisfied in a real magnetic cloud. The fact that the magnetic field strength in the front half of the magnetic cloud is higher than predicted might be due to an interaction of the magnetic cloud with slower moving material ahead of it. Such an interaction would compress the magnetic field without necessarily changing the helical configuration of the magnetic field lines inside the magnetic cloud. In any case, the constant alpha solution is at least as good as the solution of Marubashi.
The data for the December 29-30, 1967, event are shown in Figure 6 together with a constant alpha force-free magnetic field solution. Again the parameters are the same as those used by Marubashi: $\theta_{0}=-60^{\circ}, \phi_{0}=135^{\circ}, Y=0.15$, and the magnetic field is right handed. The agreement between the observed profile of the magnetic field direction and the profile for the constant alpha force-free magnetic field is very good. The model does not give a good fit to the magnetic field strength profile, although the field strength is maximum where it should be in relation to the direction profile. Apparently, whatever factor was responsible for the distortion of the magnetic field strength profile did not greatly alter the pattern of helical magnetic field lines.

Let us now consider the observations of two more magnetic clouds, one with axis nearly parallel to the ecliptic and the other with axis normal to the ecliptic. Figure 7 shows a constant alpha force-free solution for the former case, with $\theta_{0}=20^{\circ}, \phi_{0}=120^{\circ}, Y_{0}=-0.5$, and right-handed orientation. The resulting magnetic profiles are very similar to those in Figure $3 a$, as one might expect. The parameters were chosen to model the observations of the magnetic cloud which moved past Earth on March 19, 1980, which are shown in the right panel of Figure 7. The constant alpha force-free model 


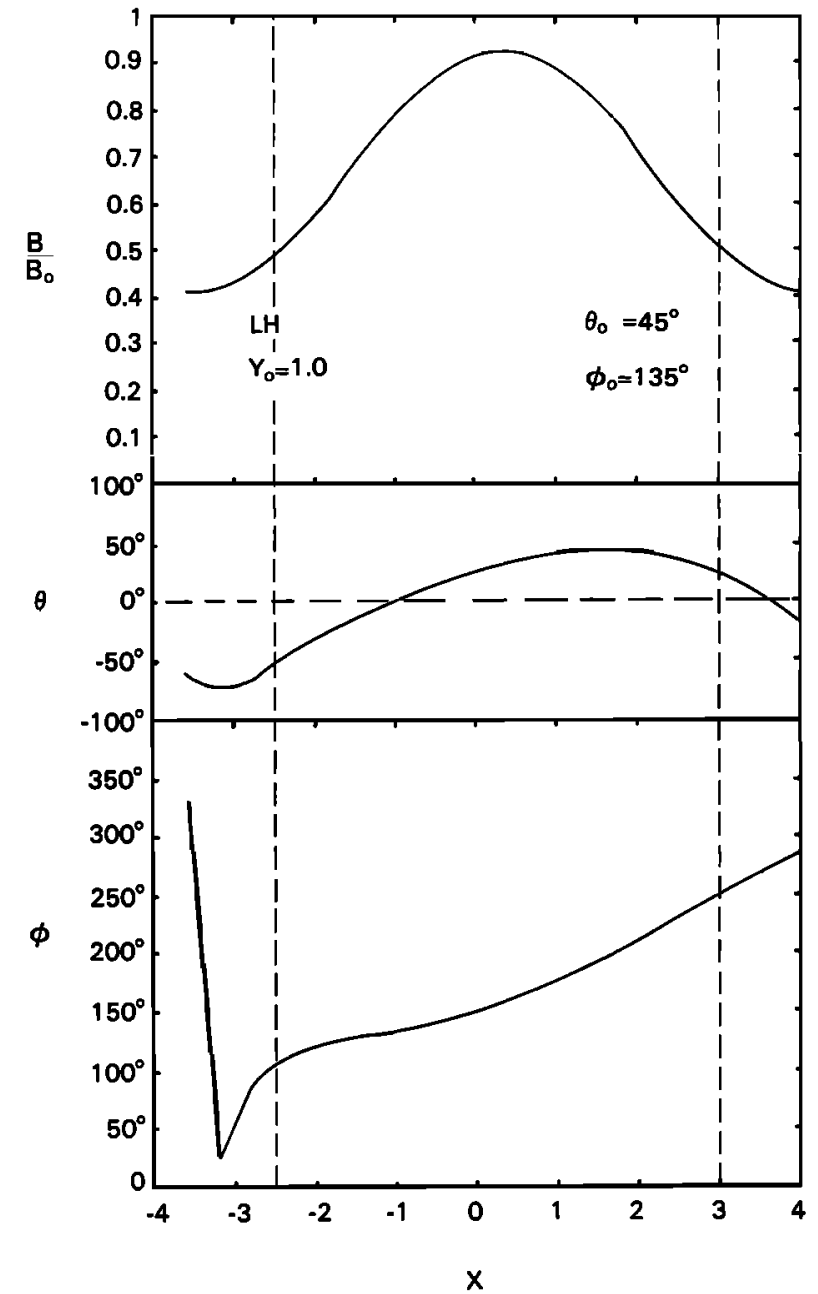

Fig. 4. The theoretical magnetic field profile which would be observed for a left-handed magnetic cloud whose axis is inclined $45^{\circ}$ with respect to the ecliptic and whose projection in the ecliptic is inclined $45^{\circ}$ with respect to the Earth-Sun line (see Figure 2).

again provides a very satisfactory description of the variations in magnetic field direction, and it gives high magnetic field strength in the magnetic cloud as observed, but it does not account for the asymmetry of the magnetic field strength profile.

Finally, we consider observations of a magnetic cloud on August 27, 1978 (Figure 8), which are unlike the magnetic clouds that have been reported in the literature in that there is no monotonic variation of the magnetic field direction from north to south or vice versa. The magnetic field profile of the data in Figure 8 resembles that in Figure $3 b$, which describes a magnetic cloud whose axis is normal to the ecliptic. Choosing $\theta_{0}=-80^{\circ}, \phi_{0}=85^{\circ}, Y_{0}=1$, and right-handed orientation, one obtains the profiles shown on the left in Figure 8. Once again one obtains a very good description of the variations in magnetic field direction found in the observations, but the magnetic field strength profile shows an asymmetry that suggests an interaction between the magnetic cloud and the flow ahead of it and fluctuations which might be related to filaments with nonnegligible plasma pressure.

\section{Summary and Discussion}

It was shown that the kinds of variations of the magnetic field direction which are observed when a magnetic cloud moves past a spacecraft at $1 \mathrm{AU}$ can be reproduced by a model of a cylindrically symmetric constant alpha force-free magnetic field. The observed magnetic field profiles depend on the position and orientation of the axis of the magnetic cloud. The canonical magnetic cloud signature, a monotonic variation of the magnetic field direction from south to north (or vice versa), is obtained for a magnetic cloud whose axis is nearly parallel to the ecliptic. We presented observations of one magnetic cloud whose axis is nearly perpendicular to the ecliptic; the signature differs from that which is most commonly observed, but it can be reproduced by the same constant alpha force-free magnetic field model. Locally, the magnetic field lines form a family of helices as in a magnetic flux rope.

Although Lundquist's solution for a constant alpha forcefree magnetic field unifies a large set of observations of magnetic clouds and provides information about the local orientation and size of magnetic clouds, there are limits to the applicability of the solution. A means of obtaining an optimal fit of Lundquist's solution data and a measure of the fit are needed.

The force-free magnetic field configurations discussed here account for the fact that the magnetic field strength is generally higher inside the magnetic cloud than near its outer boundary, but they do not account for the asymmetries and fluctuations in the magnetic field strength profile that are observed. Thus additional factors must be considered in order to model magnetic clouds more accurately. An asymmetry in the magnetic field strength profile would result from either the interaction of a fast magnetic cloud with slower material ahead of it or the interaction of a fast stream which is overtaking a magnetic cloud. Fluctuations in the magnetic field strength might be associated with irregularities in the distribution of plasma inside the magnetic cloud which might contribute significantly to the total pressure in some parts of the magnetic cloud.

Little is known about the boundaries of magnetic clouds. In order to establish the boundary conditions, which are needed for theoretical studies, it will be necessary to determine the structure of the boundaries using high-resolution data and to determine the relation between the pressure of the plasma outside the magnetic cloud and the pressure inside the magnetic cloud.

Although this paper models magnetic clouds at $1 \mathrm{AU}$ as static structures, it is known that magnetic clouds expand as they move away from the Sun. Since magnetic clouds are larger than the Sun, they must expand during their transit from the Sun to $1 \mathrm{AU}$. The velocity profile in some magnetic clouds is also indicative of expansion [Klein and Burlaga, 1982]. Evidence for continued expansion beyond $1 \mathrm{AU}$ was presented by Burlaga and Behannon [1982]. Although the expansion near the Sun is likely to be dynamical, it is possible that expansion in the interplanetary medium is basically a geometrical effect as a consequence of the motion of a magnetic cloud radially outward in a spherically expanding flow. A static force-free configuration would be an appropriate model in such a circumstance if the time to relax to the force-free configuration is less than the expansion time. There is a need for a study of the stability of a constant alpha force-free field for perturbations of this sort.

It must be emphasized that the assumption of cylindrical symmetry is an approximation that can only be valid locally. 

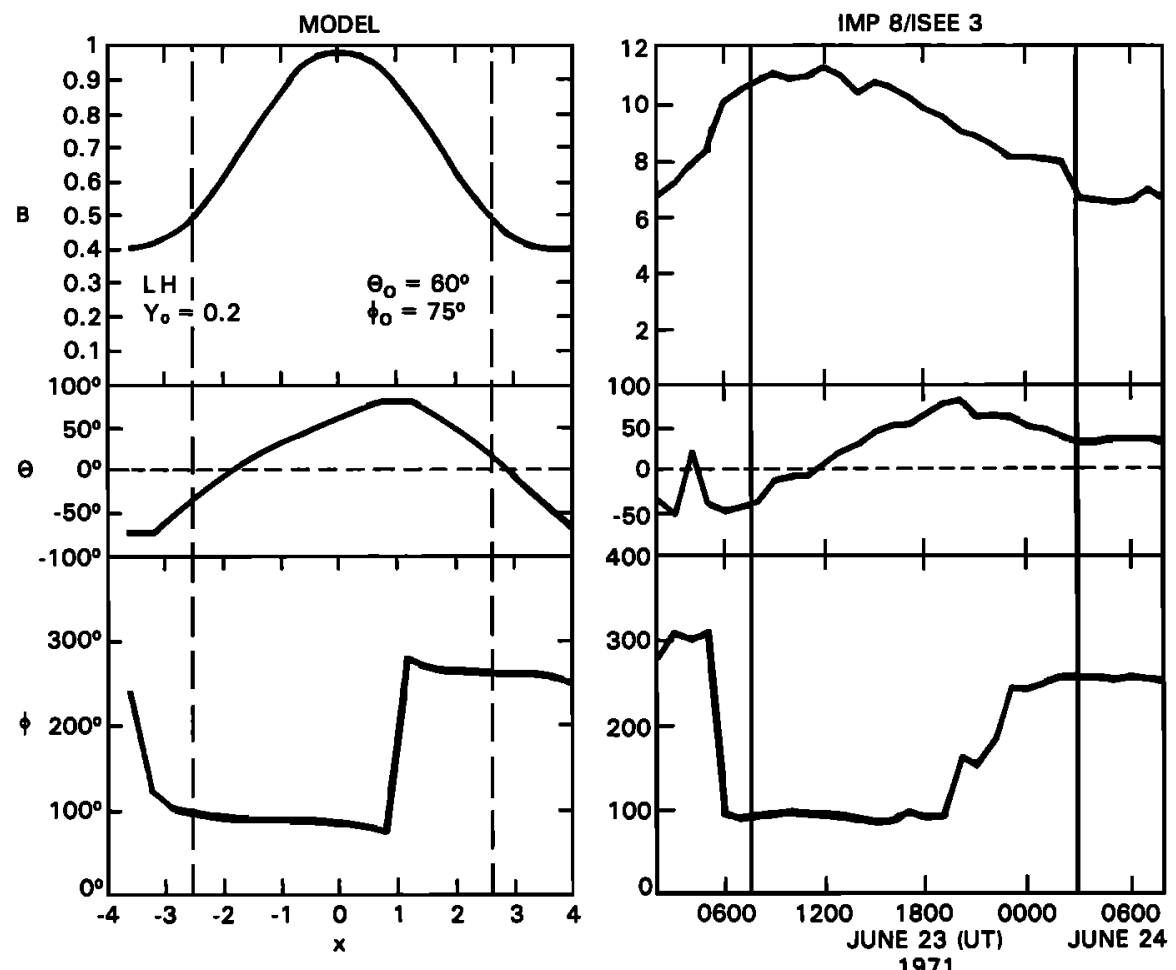

Fig. 5. Theoretical magnetic field profile (left) and observed magnetic field profile (right) for the magnetic cloud which was observed on June 23, 1971.

The global configuration of a magnetic cloud is still a mystery. One can imagine many possibilities: the ends of the cylinder might be anchored at the Sun forming loops; there might be no ends, the cylinder being closed to form a torus which is possibly oriented with its symmetry axis in the ecliptic like a smoke ring coming from the Sun; or the ends might somehow pinch off at two singular points to form a banana-shaped object. Obviously, speculations about the global configurations of magnetic clouds need to be constrained by measurements from two or more spacecraft and a better understanding of how magnetic clouds are formed.

Nothing is known about the formation of magnetic clouds.
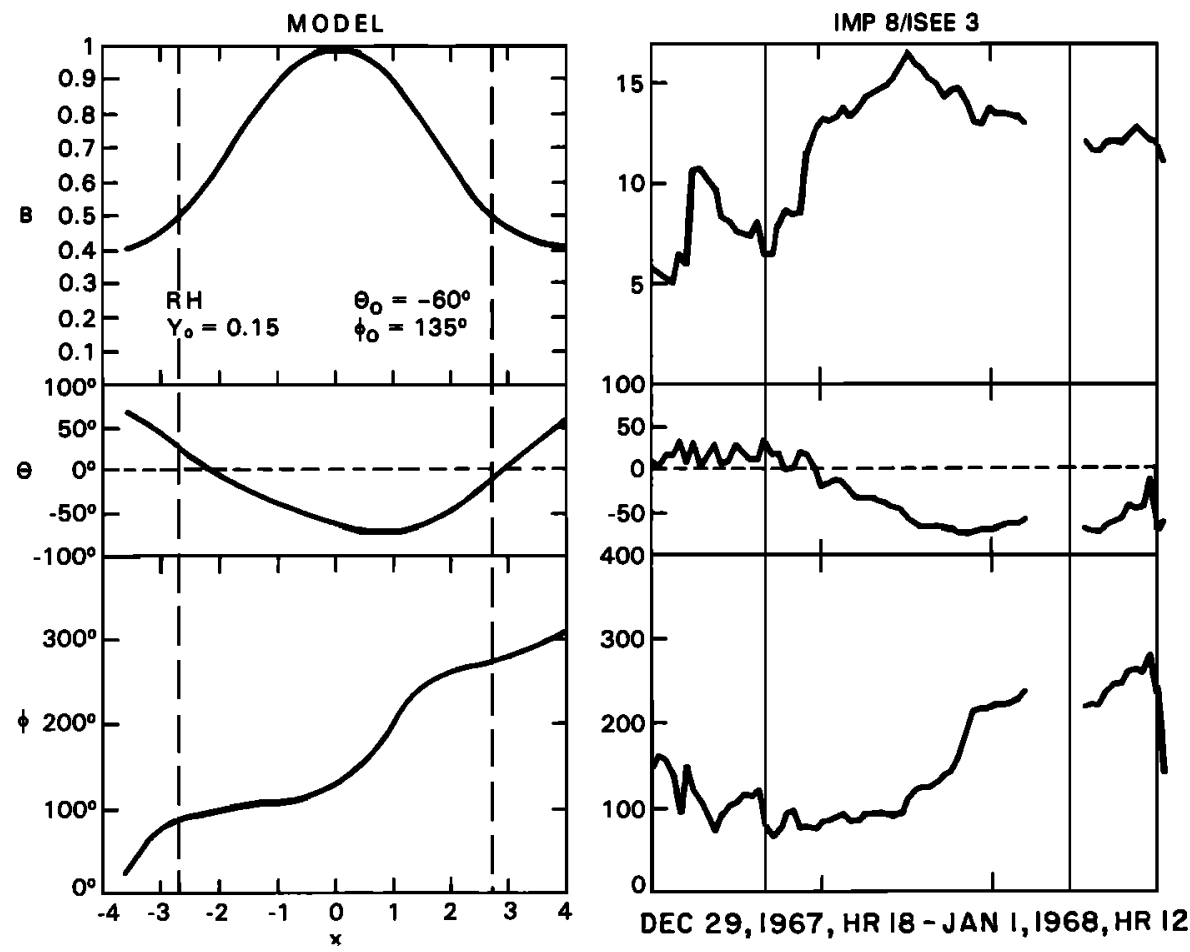

Fig. 6. Theoretical magnetic field profile (left) and observed magnetic field profile (right) for the magnetic cloud which was observed on December 29, 1967, to January 1, 1978. 

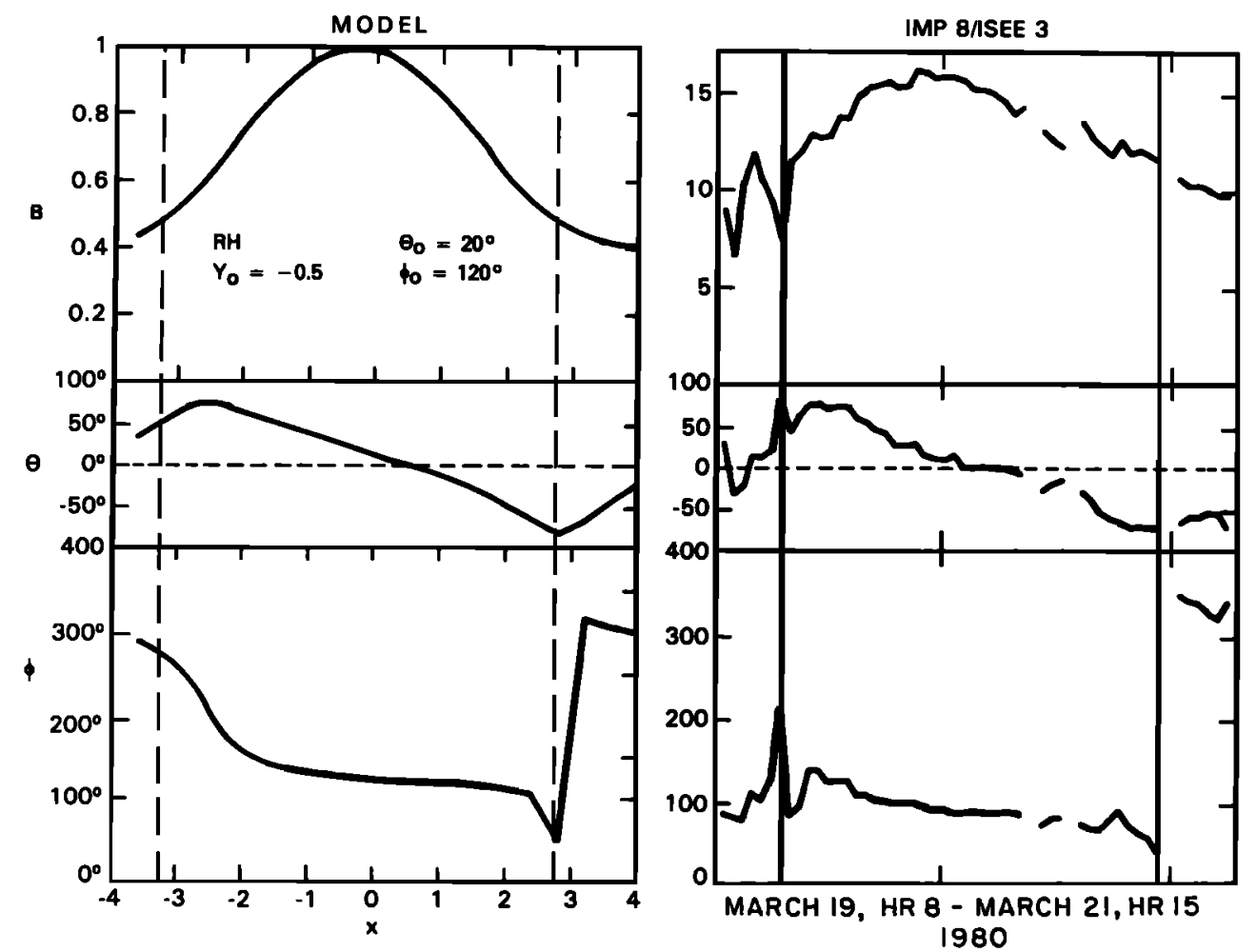

Fig. 7. Theoretical magnetic field profile (left) and observed magnetic field profile (right) for the magnetic cloud which was observed by IMP 8 and ISEE 3 on March 19-20, 1980.

On the one hand, one can imagine that a magnetic cloud is produced by the expansion or release (by reconnection) of a flux rope in the solar atmosphere. On the other hand, a magnetic cloud might result from turbulent relaxation of a discharge in a pinch configuration. These models could be investigated using the techniques already published. Other possibilities for the formation of magnetic cloud should also be considered.
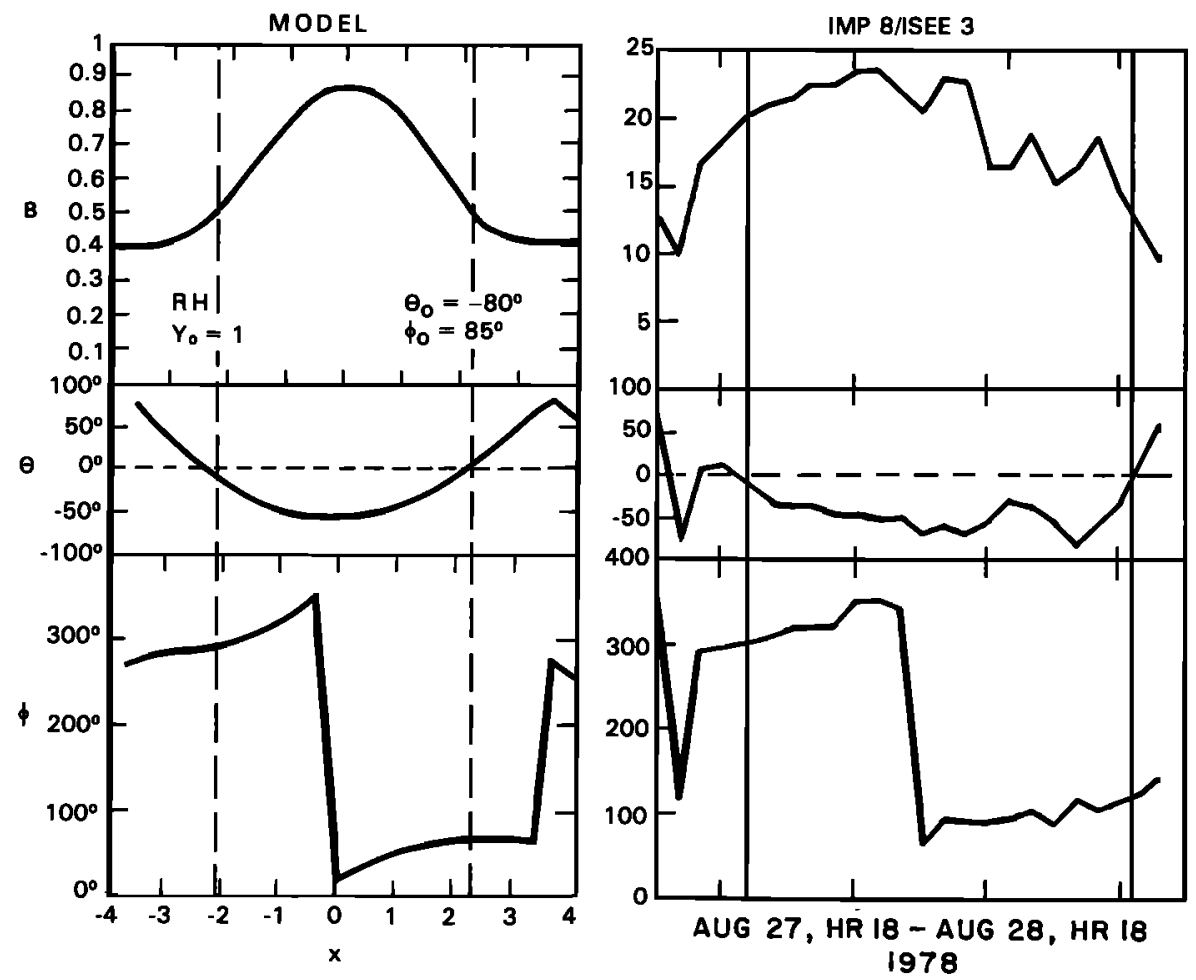

Fig. 8. Theoretical magnetic field profile (left) and observed magnetic field profile (right) for the magnetic cloud which was observed by IMP 8 and ISEE 3 on August 27-28, 1978. 
Acknowledgments. The Editor thanks L. Turner and R. Wolfson for their assistance in evaluating this paper.

\section{REFERENCES}

Buck, G. J., Force-free magnetic field solution in toroidal coordinates, J. Appl. Phys., 36, 2231, 1965.

Burlaga, L. F., MHD processes in the outer heliosphere, Space Sci. Rev., 39, 255, 1984.

Burlaga, L. F., and K. W. Behannon, Magnetic clouds: Voyager observations between 2 and 4 AU, Sol. Phys., 81, 181, 1982.

Burlaga, L. F., E. Sittler, F. Mariani, and R. Schwenn, Magnetic loop behind an interplanetary shock: Voyager, Helios, and IMP 8 observations, J. Geophys. Res., 86, 6673, 1981.

Chandrasekhar, S., and P. C. Kendall, On force-free magnetic fields, Astrophys. J., 126, 457, 1957.

Chandrasekhar, S., and L. Woltjer, On force-free magnetic fields, Proc. Natl. Acad. Sci. U.S.A., 44, 285, 1958.

Dahlburg, J. P., D. Montgomery, G. D. Doolen, and L. Turner, Turbulent relaxation to a force-free field-reversed state, Phys. Rev. Lett., 57, 428, 1986.

Elphic, R. C., and C. T. Russell, Magnetic flux ropes in the Venus ionosphere: Observations and models, J. Geophys. Res., 88, 58, 1983.

Ferraro, V. C. A., and C. Plumpton, Magneto-Fluid Mechanics, Oxford University Press, New York, 1966.

Field, G., Magnetic helicity in astrophysics, in Magnetospheric Phenomena in Astrophysics, edited by R. I. Epstein and W. C. Feldman, American Institute of Physics, New York, 1986.

Goldstein, H., On the field configuration in magnetic clouds, Solar Wind Five, NASA Conf. Publ., 2280, 731, 1983.

Heyvaerts, J., and E. R. Priest, Coronal heating by reconnection in D.C. current systems: A theory based on Taylor's hypothesis, Astron. Astrophys., 137, 163, 1984.

Hood, A. W., MHD instabilities, in Solar System Magnetic Fields, edited by E. R. Priest, p. 80. D. Reidel, Hingham, Mass., 1985.

Ivanov, K. G., and A. F. Harshiladze, Dynamics of hydromagnetic clouds from powerful solar flares, Sol. Phys., 92, 351, 1984.
Klein, L. W., and L. F. Burlaga, Interplanetary magnetic clouds at 1 AU, J. Geophys. Res., 87, 613, 1982.

Konigl, A., and A. Choudhur, Force-free equilibria of magnetized jets, Astrophys. J., 289, 173, 1985.

Lundquist, S., Magnetohydrostatic fields, Ark. Fys., 2, 361, 1950.

Lust, R., and A. Schluter, Force-free magnetic fields, Z. Astrophys., 34, 353, 1954.

Marubashi, K., Structure of the interplanetary magnetic clouds and their solar origins, Adv. Space Res., 6, 1, 1986.

Moffatt, H. K., Magnetic Field Generation in Electrically Conducting Fluids, p. 28, Cambridge University Press, New York, 1978.

Parker, E. N., The gross dynamics of a hydromagnetic gas cloud, Astrophys. J. Suppl. Ser., 25, 51, 1957.

Suess, S. T., Magnetic clouds and the pinch effect, J. Geophys. Res., 93, 5437, 1988.

Taylor, J. B., Relaxation of toroidal plasma and generation of reverse magnetic fields, Phys. Rev. Lett., 33, 1139, 1974.

Taylor, J. B., Relaxation of toroidal discharges, in Pulsed High Beta Plasmas, edited by D. E. Evans, p. 64, Pergamon, New York, 1976.

Taylor, J. B., Relaxation and magnetic reconnection in plasmas, Rev. Mod. Phys., 58, 741, 1986.

Turner, L., Is magnetic energy the source of synchrotron radio emission in jets?, Astrophys. J., 305, 668, 1986.

Turner, L., and S. C. Prager, Magnetic topology of a helical ohmic steady state, Phys. Fluids, 30, 787, 1987.

Voslamber, D., and D. K. Callebaut, Stability of force-free magnetic fields, Phys. Rev., 128, 2016, 1962.

Woltjer, L., A theorem on force-free magnetic fields, Proc. Natl. Acad. Sci. U.S.A., 44, 389, 1958.

L. F. Burlaga, Laboratory for Extraterrestrial Physics, Code 692, NASA Goddard Space Flight Center, Greenbelt, MD 20771.

(Received September 18, 1987; revised February 1, 1988; accepted February 3, 1988.) 